\title{
Entanglement Entropy in an Antiferromagnetic Heisenberg Spin Chain with Boundary Impurities
}

\author{
Jie Ren ${ }^{1 *}$, Shiqun $\mathrm{Zhu}^{1 \dagger}$, and Xiang $\mathrm{Hao}^{2}$ \\ ${ }^{1}$ School of Physical Science and Technology, Suzhou University, \\ Suzhou, Jiangsu 215006, People's Republic of China and \\ ${ }^{2}$ Department of Physics, Suzhou University of Science and Technology, \\ Suzhou, Jiangsu 215011, People's Republic of China
}

\begin{abstract}
The effects of boundary impurities on the entanglement entropy in an antiferromagnetic Heisenberg opened spin-1/2 chain are investigated. The method of density-matrix renormalization-group is used to obtain the bipartite entanglement. The entropy increases when the length of the subsystem increases. It will approach to a constant when system length is very large. With the same impurity interaction, qutrit impurities of spin-1 can increase the entanglement entropy.
\end{abstract}

PACS numbers: 03.67.Mn, 03.65.Ud, 05.50.+q, 75.10.Jm

Key words: Entropy, Boundary Impurities, DMRG

\footnotetext{
* E-mail: jren@cslg.edu.cn

$\dagger$ Corresponding author, E-mail: szhu@suda.edu.cn
} 


\section{INTRODUCTION}

Recently, the entanglement has been recognized as an important resource of some quantum mechanical phenomena, such as quantum teleportation, quantum cryptography, quantum computation, and violation of Bell's inequality [1-3]. Many investigations show that entanglement exists naturally in the spin chain when the temperature of system is at zero. A useful many-body entanglement measure of a pure state is the von Neumann entanglement entropy [4], which can also quantify quantum phase transition [5]11]. The bipartite entanglement in systems of atomic Bose-Einstein condensate was studied [12, 13]. The entanglement entropy in the antiferromagnetic Heisenberg $X X$ chain and Ising model was investigated [14]. In isotropic antiferromagnetic Heisenberg model, the universal form of the entropy is predicted by [15]

$$
S_{L}=\frac{c}{3} \log _{2} L+k
$$

where $c$ is the central charge and $k$ is a non-universal constant. For a spin chain of open boundary condition, the analogous formula $c / 3$ should be replaced by $c / 6$ for a part of length $L$ in an infinite one-dimension system [16, 17]. The analogous formula is dependent on the boundary conditions of the block and the rest of the chain. If the block has two boundaries with the rest of the chain then the factor is $c / 3$, while if the block has just one boundary as in the case of a block consisting of the first adjacent spins of a semi-infinite chain, then the factor is $c / 6$. Recently, it is shown that a feeble central bound defect [18] or single impurity in the boundary [19] has strong influence on the entropy, though the entropy measures the mutual coupling of the two parts of a system in wave function. A weak transverse boundary magnetic field impurity [20] and domain walls [21] generated by antiparallel magnetic field have different effects on the entanglement entropy. Moreover, impurities show their strong influences on spin correlation function [22, 23]. It would be interesting to investigate the effects of boundary impurities on the entanglement entropy in an opened antiferromagnetic Heisenberg spin chain.

In this paper, the entanglement entropy of a spin $-1 / 2$ antiferromagnetic Heisenberg chain with boundary impurities located at two ends is investigated. In scetion II, the Hamiltonian of an antiferromagnetic Heisenberg spin-1/2 chain is presented. By using the method of

density-matrix renormalization-group (DMRG) [24, 25], the entropy of ground state is cal- 
culated and the effects of the impurities are analyzed in section III. A discussion concludes the paper.

\section{HAMILTONIAN OF HEISENBERG SPIN CHAIN}

The Hamiltonian of a spin-1/2 Heisenberg opened chain with boundary impurities at two ends can be written as

$$
H=\sum_{i=2}^{N-2} J \vec{S}_{i} \vec{S}_{i+1}+\alpha\left(\vec{S}_{1} \vec{S}_{2}+\vec{S}_{N-1} \vec{S}_{N}\right)
$$

Where the coupling exchange $J>0$ corresponds to the antiferromagnetic case, $\vec{S}_{j}$ are spin operators, $N$ is the length of the spin chain. The coupling exchange $\alpha$ is impurity interaction. For simplicity, $J=1$ is assumed in this paper.

The entropy is used as a measure of the bipartite entanglement. If $|G s\rangle$ is the ground state of a chain of $\mathrm{N}$ qubits, a reduced density matrix of $L$ contiguous qubits can be written as

$$
\rho_{L}=\operatorname{Tr}_{N-L}|G s\rangle\langle G s|
$$

The bipartite entanglement between the right-hand $L$ contiguous qubits and the rest of the system can be measured by the entropy

$$
S_{L}=-\operatorname{Tr}\left(\rho_{L} \log _{2} \rho_{L}\right)
$$

One of the properties of the entropy of a block of the system can be given by

$$
S_{L}=S_{N-L}
$$

since the spectrum of the reduced density matrix $\rho_{L}$ is the same as that of $\rho_{N-L}$.

\section{ENTANGLEMENT OF ENTROPY WITH IMPURITIES}

In order to calculate the entropy accurately using the method of DMRG, the length of the spin chain needs to be relatively long. The length of the spin chain is chosen to be $N=256$. The total number of the density matrix eigenstates held in the system block is $m=128$ in the basis truncation procedure. 
To check the accuracy of the results from the method of DMRG, the open boundary condition without impurities of $\alpha=1$ is considered. The corresponding results of finite spin chain, which is predicted by conformal field theory(CFT), can be considered as a benchmark. It can be written as

$$
S_{L}=\frac{c}{6} \log _{2}\left[\frac{N}{\pi} \sin \left(\frac{\pi}{N} L\right)\right]+A,
$$

where $c$ is the central charge and $A$ is a non-universal constant [26, 27]. There are large oscillations between even and odd $L$-value entropy. To avoid these relatively large oscillations, the even-value entropy is chosen. The entropy $S_{L}$ between contiguous $L$ qubits and the remain $N-L$ qubits is plotted as a function of the subsystem L in Fig. 1 when $N=160,200$ and 256. For $L<8$, the results of DMRG are slightly lower than that of CFT. For $L>8$, almost perfect agreement between the two results is obtained. For different values of $N$ with large $L$, $S_{L}$ is small for small values of $N$. For small $L$, there is almost no difference between different values of $N$. It seems that $S_{L}$ approaching a constant for very large $L$ is mainly due to finite size effect. The entropy $S_{L}$ is also plotted as a function of $\log _{2}\left[\frac{N}{\pi} \sin \left(\frac{\pi}{N} L\right)\right]$ in the inset of Fig. 1. It is shown that the entropy appears as a straight line whose slope is very close to $c / 6$.

The entanglement entropy $S_{L}$ is plotted as a function of the subsystem length $L$ for different values of the impurity interaction $\alpha$ in Fig. 2(a). It is seen that the entropy $S_{L}$ increases with the subsystem length $L$ and then approaches a constant when $L$ is very large for $\alpha=0.1,2.0$. When $\alpha=0.3,0.5$, the entropy $S_{L}$ decreases slightly, then increases and approaches a constant for very large $L$. The minimal value is 1.53 at $L=6$ for $\alpha=0.3$ and 1.16 at $L=4$ for $\alpha=0.5$. When $\alpha=0.1, S_{L}$ approaches the value about 2.4 for very large $L$. While for $\alpha=0.3,0.5,2.0, S_{L}$ approaches the value about 1.6 for very large $L$. The influence of the impurity at two ends of the Heisenberg spin-1/2 chain depends on the value of the impurity interaction $\alpha$. For $\alpha=\alpha_{0}=0.235$, the strength alternation of even bond and odd bond in the center of the spin chain is minimized to almost close to zero [24]. For $\alpha<\alpha_{0}$, the even sublattice is favored. This induces larger value of $S_{L}$. While for $\alpha>\alpha_{0}$, the odd sublattice is favored. This induces smaller value of $S_{L}$. If the value of $\alpha$ is less than $\alpha_{0}=0.235$, the effects of the impurity on the entanglement entropy $S_{L}$ is stronger. Therefore, the entanglement entropy $S_{L}$ of $\alpha=0.1$ is much larger than the $S_{L}$ of $\alpha=0.3,0.5$ and $2.0[18,19,24]$. The entropy $S_{L}$ is also plotted as a function of $\log _{2}\left[\frac{N}{\pi} \sin \left(\frac{\pi}{N} L\right)\right]$ in the inset of Fig. 2(a). It is seen that $S_{L}$ is almost a straight as a function of $\log _{2}\left[\frac{N}{\pi} \sin \left(\frac{\pi}{N} L\right)\right]$ for 
very large $L$.

If $S_{L \alpha}$ is the entropy with impurities at two ends and $S_{L 0}$ is the entropy without impurities, the difference of the entropy $\Delta S_{L}$ can be defined as

$$
\Delta S_{L}=S_{L \alpha}-S_{L 0} .
$$

The entropy difference $\Delta S_{L}$ may also be called "impurity entanglement entropy" that is induced by adding impurities at two ends of the spin chain [19]. The entropy difference $\Delta S_{L}$ is plotted as a function of the subsystem length $L$ for different values of the impurity interaction $\alpha$ in Fig. 2(b). It is shown that $\Delta S_{L}$ decreases and then approaches a constant when the subsystem $L$ increases for $\alpha=0.1,0.3,0.5$. The value of $\Delta S_{L}$ increases and then approaches a constant with increase of the subsystem length $L$ when $\alpha=2.0$. It seems that the effect of impurity decreases with the increase of the subsystem $L$ when $\alpha<\alpha_{0}=1.0$. However, the effect of the impurity increases when $\alpha>\alpha_{0}=1.0$. The entropy difference $\Delta S_{L}$ is also plotted as a function of $\log _{2}\left[\frac{N}{\pi} \sin \left(\frac{\pi}{N} L\right)\right]$ in the inset of Fig. 2(b). It is seen that $\Delta S_{L}$ is almost a straight as a function of $\log _{2}\left[\frac{N}{\pi} \sin \left(\frac{\pi}{N} L\right)\right]$ for very large $L$. Similar to that shown in Fig. 2(a), the entropy difference $\Delta S_{L}$ of $\alpha=0.1$ is much larger than $\Delta S_{L}$ of $\alpha=0.3,0.5$ and 2.0. This is mainly due to the fact that small value of $\alpha<\alpha_{0}=0.235$ can induce stronger effect of impurity on $\Delta S_{L}$ since the even sublattice is favored [18, 19, 24].

From Fig. 2, it is seen that both values of $S_{L}$ and $\Delta S_{L}$ are decreases when $\alpha$ increases especially for small number of $L$. There are large differences of $S_{L}$ and $\Delta S_{L}$ for different impurity interactions $\alpha$ when $L$ is small. If $L$ is quite large, $S_{L}$ and $\Delta S_{L}$ approach to constants. If $\alpha=0.3,0.5$ and $2.0, S_{L}$ approaches to about 1.6 while $\Delta S_{L}$ approaches to about zero for quite large $L$. It seems that the effect of the impurity at two ends is very small for large $L$. If $\alpha=0.1$, both $S_{L}$ and $\Delta S_{L}$ are quite large. It seems that the small value of impurity interaction $\alpha<\alpha_{0}=0.235$ can induce strong effect on $S_{L}$ and $\Delta S_{L}$.

The central charge $c$ in Eq. (6) plays an important role in the measurement of the entanglement entropy. The central charge $c$ can be calculated numerically by [17, 19]

$$
c(L)=6\left[\frac{S_{L+2}-S_{L-2}}{T(L+2)-T(L-2)}\right],
$$

where

$$
T(L)=\log _{2}\left[\frac{N}{\pi} \sin \left(\frac{\pi}{N} L\right)\right] .
$$


The central charge labeled by $c(L)$ is plotted in Fig. 3(a) as a function of the subsystem length $L$ for different values of the impurity interaction $\alpha$. When $\alpha=0.1$, the central charge $c(L)$ increases to a peak and then decreases slowly with the increase of the subsystem length $L$. The central charge $c(L)$ decreases and then approaches a constant with the increase of the subsystem $L$ when $\alpha=2.0$. When $\alpha=0.3,0.5$, the central charge $c(L)$ increases and almost approaches a constant with the increase of the subsystem length $L$. The central charges of $c(L=6)$ and $c(L=4)$ are negative when $\alpha=0.3$ and 0.5 respectively. This corresponds to the minimum values of $S_{L}$ shown in Fig. 2(a). For $\alpha>0.235$, the central charge $c(L)$ increases with increasing value of $\alpha$. For $\alpha=0.1<0.235$, c(L) of $\alpha=0.1$ is larger than that of $\alpha=0.3$, it decreases and finally approaches to that of $\alpha=0.3$ when $L$ is very large. The value of $c(L)$ of $\alpha=0.1$ is larger than that of $\alpha=0.5$ if $L<20$. If $L>20, c(L)$ of $\alpha=0.1$ is smaller than that of $\alpha=0.5$. This is mainly due to the stronger singlet bonds on the even numbered links of the chain for $\alpha<0.235$ [24]. Since the central charge may clarify the behavior of the entropy for large values of subsystem, the central charge $c(L=80)$ is plotted as a function of impurity interaction $\alpha$ in Fig. 2(b) when $1 \ll L(=80)<N / 2(=128)$. It is seen that the central charge $\mathrm{c}$ reaches a minimum value when impurity interaction $\alpha=0.235$. When impurity interaction $\alpha<0.235$, the central charge $c$ decreases with impurity interaction $\alpha$ increases. The central charge $c$ increases with impurity interaction $\alpha$ increases when $\alpha>0.235$. It approaches to 1.0 when impurity interaction $\alpha$ is close to 2.0 .

If the impurities are qutrit with spin-1 operators $\overrightarrow{S^{\prime}}$, the effects of qutrit-impurities on the entropy can also be investigated. The entanglement entropy $S_{L}$ and the difference of the entropy $\Delta S_{L}$ are plotted in Figs. 4(a) and 4(b) respectively as a function of the subsystem length $L$ for impurity interaction $\alpha$ and different impurities. The entropy $S_{L}$ and the difference of the entropy $\Delta S_{L}$ are also plotted as a function of $\log _{2}\left[\frac{N}{\pi} \sin \left(\frac{\pi}{N} L\right)\right]$ in the insets of Figs. 4(a) and 4(b). Similar to that shown in Fig. 2, both $S_{L}$ and $\Delta S_{L}$ of spin-1 decreases with the increase of $\alpha$. The entropy $S_{L}$ increases and then almost approaches a constant when the the subsystem length $L$ increases. The entropy of impurity with spin- 1 is much larger than that with spin- $1 / 2$. For the impurity of spin- $1 / 2$, the entropies of $\alpha=0.5$ and $\alpha=2.0$ are almost not distinguishable when the subsystem length $L$ is very large. While for the impurity of spin-1, the difference of the entropies of $\alpha=0.5$ and $\alpha=2.0$ are quite large 
and approach a constant when $L$ is very large. It is clear that the effects of qutrit impurity on the entanglement entropy are much stronger than that of qubit impurity. It seems that it is more easily to control the entropy of the system using qutrit impurity.

\section{DISCUSSION}

It is clear that the impurity interaction and the impurity spin have a strong influence on the entanglement of the two subsystems [19, 28, 29]. For pairwise entanglement between the impurity spin and the spin chain, the two boundary spins will have a strong tendency to form a singlet pair when the impurity interaction is large. This will reduce the entanglement between the boundary of the two spin subsystems and the rest of the system. The value of entanglement entropy is mainly determined by the density-matrix spectra, extremely by the few largest eigenvalues of the reduced density matrix [18, 20, 25]. For qubit impurities, impurities can affect the entropy between two subsystems by changing the distribution of the reduced density-matrix spectra. If the impurities are qutrits with the same impurity interaction, not only the distribution of the reduced density-matrix spectra is changed, but also the degree of the freedom of density-matrix spectra of the subsystem is enlarged in the Hilbert space. This is similar to the result of the entropy with the increase of subsystems [15, 20, 21].

The effects of boundary impurities on the bipartite entanglement in an antiferromagnetic Heisenberg open spin chain are discussed. Using the method of density-matrix renormalization-group, entanglement entropy is calculated for the even number subsystem. The entanglement entropy decreases with the increase of the impurity interaction while it increases with the increase of the subsystem length. When the system length is very large, the entanglement entropy approaches a constant due to finite size effect. The influences of boundary impurities with qutrit of spin-1 are much stronger than that of qubit of spin-1/2. With the same impurity interaction, qutrit impurities can increase the entanglement. All the results are dependent with the selection of even subsystem. This is shown that the entropy of the system with qutrit impurity can be more easily controlled.

\section{Acknowledgements}

It is a pleasure to thank Yinsheng Ling and Jianxing Fang for their many helpful dis- 
cussions. The financial supports from the National Natural Science Foundation of China (Grant No. 10774108) and the Creative Project for Doctors of Jiangsu Province of China are gratefully acknowledged.

[1] M. A. Nielson and I. L. Chuang, Quantum Computation and Quantum Information (Cambridge University Press, Cambridge, England, 2000).

[2] C. H. Bennett, G. Brassard, C. Crepeau, R. Jozsa, A. Peres, and W. K. Wootters, Phys. Rev. Lett. 70(1993) 1895.

[3] M. Murao, D. Jonathan, M. B. Plenio, and V. Vedral, Phys. Rev. A 59(1999) 156.

[4] C. H. Bennett, D. P. DiVincenzo, J. A. Smolin, and W. K. Wootters, Phys. Rev. A 54(1996) 3824 .

[5] S. Sachdev, Quantum Phase Transitions (Cambridge University Press, Cambridge, England, 1999).

[6] S. J. Gu, S. S. Deng, Y. Q. Li, and H. Q. Lin, Phys. Rev. Lett. 93(2004) 086402.

[7] A. Anfossi, P. Giorda, A. Montorsi, and F. Traversa, Phys. Rev. Lett. 95(2005) 056402.

[8] N. Lambert, C. Emary, and T. Brandes, Phys. Rev. A 71(2005) 053804.

[9] D. Larsson and H. Johannesson, Phys. Rev. Lett. 95(2005) 196406.

[10] R. A. Molina and P. Schmitteckert, Phys. Rev. B 75(2007) 235104.

[11] Ö. Legeza, J. Sólyom, L. Tincani, and R. M. Noack, Phys. Rev. Lett. 99(2007) 087203.

[12] G. J. Milburn, J. Corney, E. M. Wright, and D. F. Walls, Phys. Rev. A 55(1997) 4318.

[13] A. P. Tonel, J. Links, and A. Foerster, J. Phys. A 38(2005) 1235.

[14] G. Vidal, J. I. Latorre, E. Rico, and A. Kitaev, Phys. Rev. Lett. 90(2003) 227902.

[15] J. I. Latorre, E. Rico, and G. Vidal, Quant. Inf. Comput. 4(2004) 48.

[16] C. Holzhey, F. Larsen, and F. Wilczek, Nucl. Phys. B 424(1994) 443.

[17] P. Calabrese and J. Cardy, J. Stat. Mech (2004) P06002 hep-th/0405152.

[18] J. Z. Zhao, I. Peschel, and X. Q. Wang, Phys. Rev. B 73 (2006) 024417.

[19] E. S. Søensen, M. S. Chang, N. Laflorencie, and I. Affleck, J. Stat. Mech (2007) P08003.

[20] H. Q. Zhou, T. Barthel, J. O. Fjærestad, and U. Schollwöck, Phys. Rev. A 74(2006) 050305(R).

[21] J. Ren, and S. Zhu, Phys. Rev. A 77(2008) 034303. 
[22] J. Lou, J. Dai, S. Qin, Z. Su, and L. Yu, Phys. Rev. B 62(2000) 8600.

[23] G. Fáth, Ö. Legeza, P. Lajkó, and F. Iglói, Phys. Rev. B 73(2006) 214447.

[24] S. R. White, Phys. Rev. B 48(1993) 10345.

[25] U. Schollwöck, Rev. Mod. Phys. 77(2005) 259.

[26] N. Laflorencie, E. S. Sørensen, M. S. Chang, and I. Affleck, Phys. Rev. Lett. 96(2006) 100603.

[27] G. D. Chiara, S. Montangero, P. Calabrese, and R. Fazio, J. Stat. Mech (2006) P03001 condmat/0512586.

[28] X. Wang, Phys. Rev. E 69 (2004) 066118.

[29] E. S. Søensen, M.S. Chang, N. Laflorencie, and I. Affleck, J. Stat. Mech (2007) L01001 condmat/0606705. 


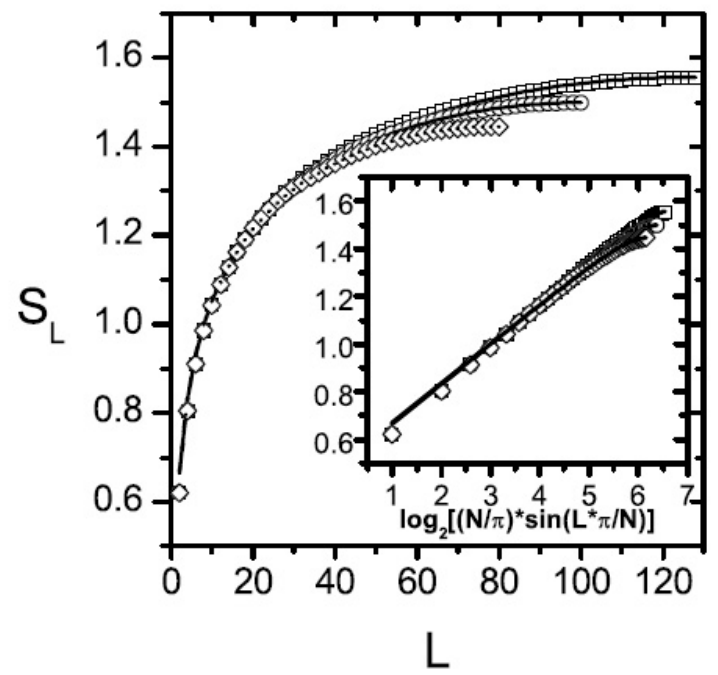

FIG. 1: The entropy $S_{L}$ between contiguous $L$ qubits and the remain $N-L$ qubits is plotted as a function of the subsystem length $L$ for $N=160,200,256$ (from bottom to top). The dashed line is obtained from CFT and the symbol $\bigcirc$ is obtained from DMRG. 

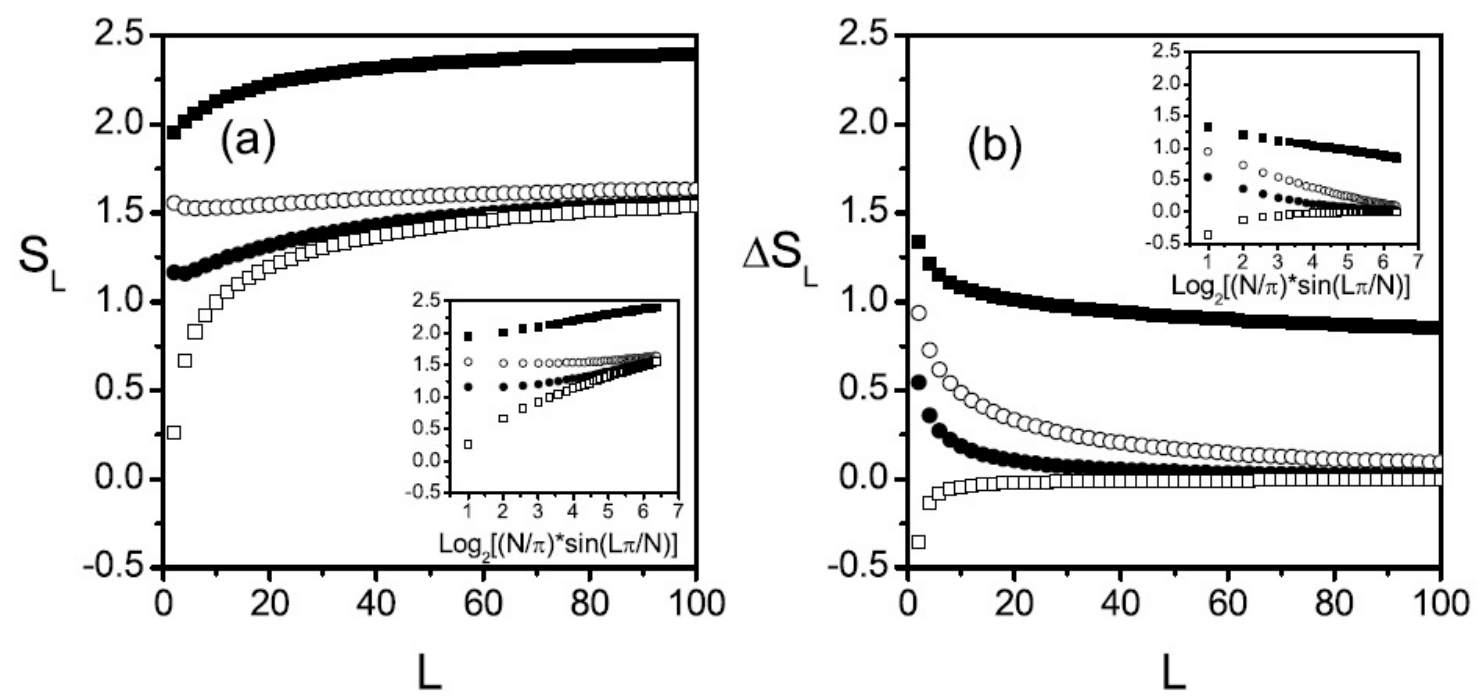

FIG. 2: The entanglement entropy $S_{L}$ and the entropy difference $\Delta S_{L}$ are plotted as a function of the subsystem length $L$ for different values of the impurity interaction $\alpha$. (a). The entropy $S_{L}$. (b). The entropy difference $\Delta S_{L}$. The symbols are for $\alpha=0.1(\mathbf{\square}), 0.3(\circ), 0.5(\bullet), 2.0(\square)$. 

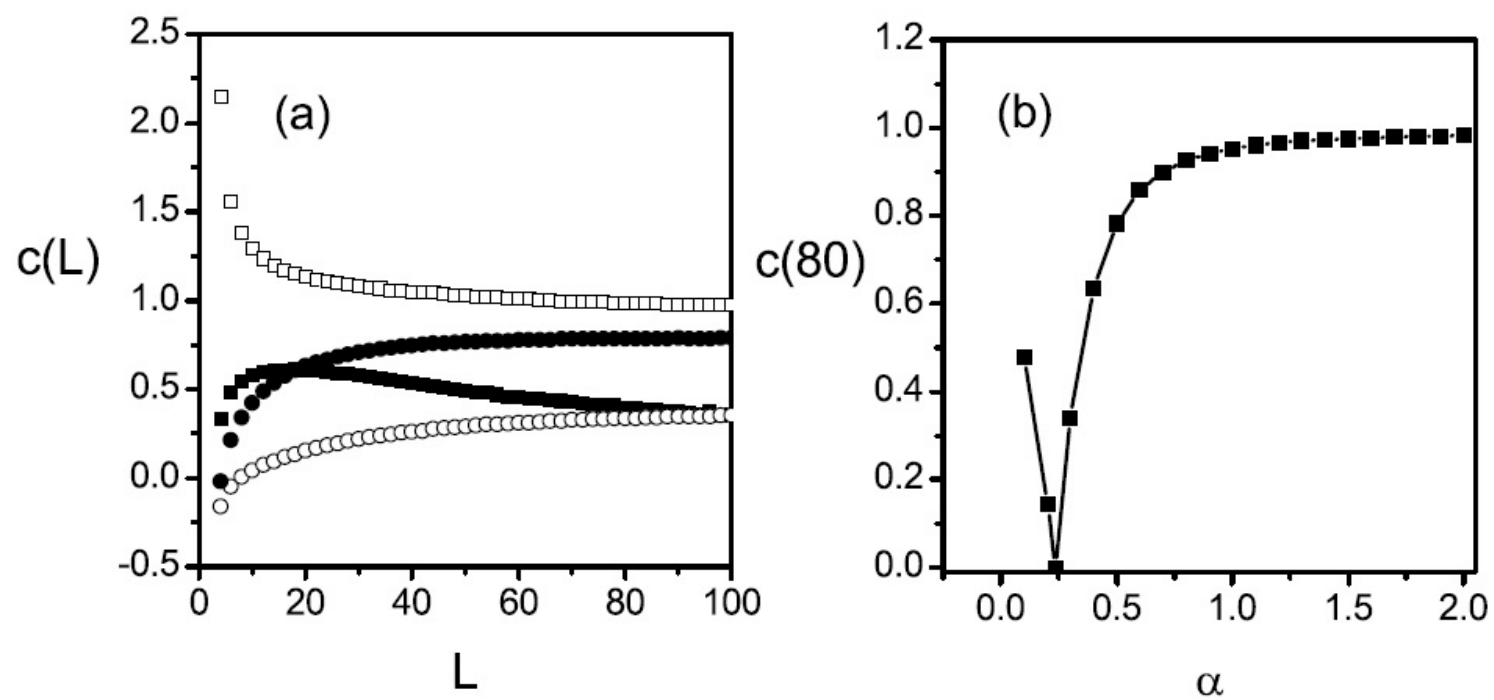

FIG. 3: (a). The central charge $c(L)$ is plotted as a function of the subsystem length $L$ for different values of the impurity interaction $\alpha$. The symbols are for $\alpha=0.1(\boldsymbol{\square}), 0.3(\circ), 0.5(\bullet), 2.0(\square)$. (b). The central charge $c(80)$ is plotted as a function of impurity interaction $\alpha$. 

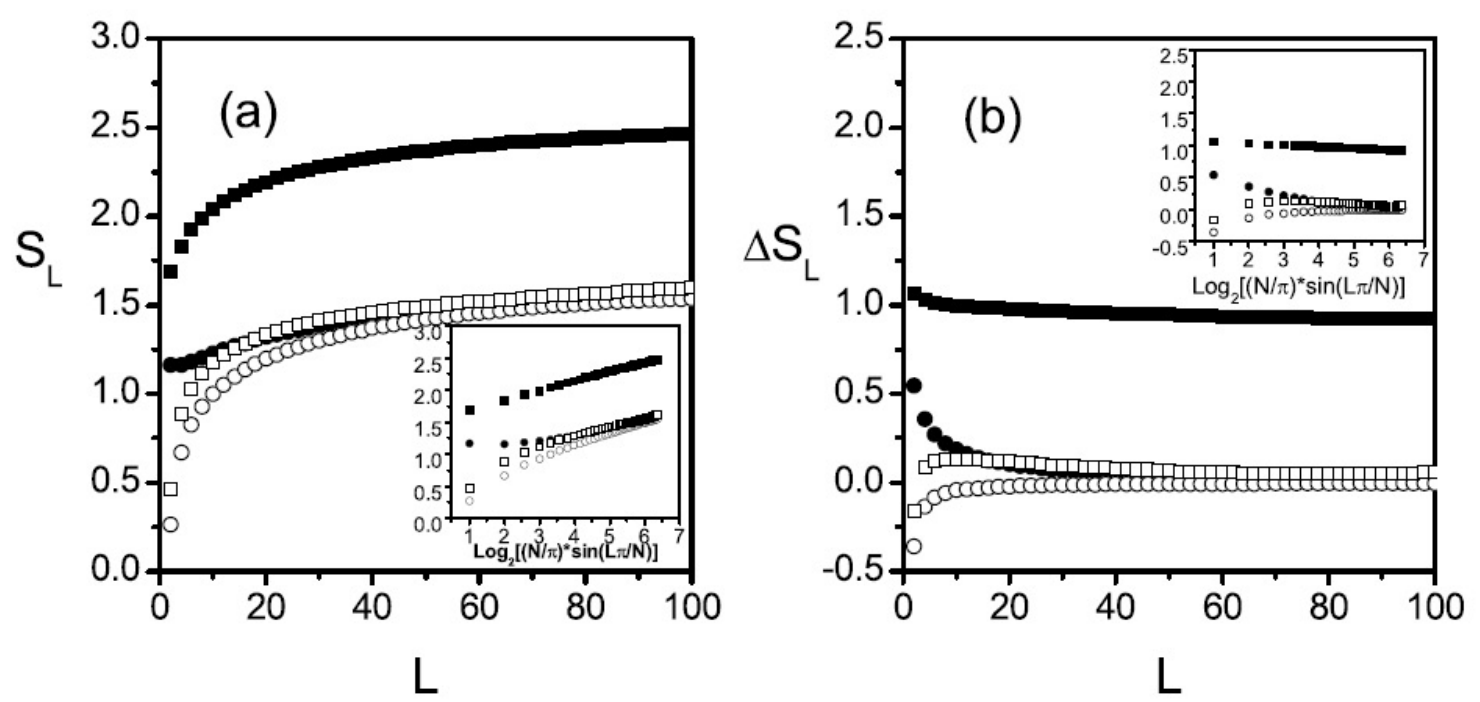

FIG. 4: The entanglement entropy $S_{L}$ and the entropy difference $\Delta S_{L}$ are plotted as a function of the subsystem length $L$ for different values of the impurity interaction $\alpha$ and the spins $1 / 2$ and 1 . (a). The entropy $S_{L}$. (b). The entropy difference $\Delta S_{L}$. The symbols are for qubit impurity with $\alpha=0.5(\bullet), 2.0(\circ)$, qutrit impurity with $\alpha=0.5(\boldsymbol{\square}), 2.0(\square)$. 